\title{
Upregulated EFNB2 and EPHB4 promotes lung development in a nitrofen-induced congenital diaphragmatic hernia rat model
}

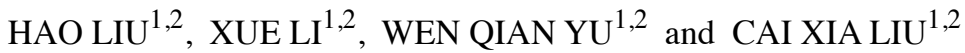 \\ ${ }^{1}$ Department of Gynecology and Obstetrics, Shengjing Hospital Affiliated to China Medical University, \\ Shenyang, Liaoning 110004; ${ }^{2}$ Key Laboratory of Maternal-Fetal Medicine of Liaoning Province, \\ Benxi, Liaoning 117004, P.R. China
}

Received February 8, 2018; Accepted August 3, 2018

DOI: $10.3892 /$ ijmm.2018.3824

\begin{abstract}
Congenital diaphragmatic hernia (CDH) is a common congenital malformation associated with high mortality rates, mainly due to pulmonary hypoplasia and persistent pulmonary hypertension following birth. The present study aimed to investigate abnormal lung development in a rat $\mathrm{CDH}$ model, and examine temporal and spatial changes in the expression of ephrin type-B receptor 4 (EPHB4) and ephrin-B2 (EFNB2) during fetal lung development, to elucidate the role of these factors during lung morphogenesis. Pregnant rats received nitrofen on embryonic day (E) 8.5 to induce $\mathrm{CDH}$, and fetal lungs were collected on E13.5, E15.5, E17.5, E19.5, and E21.5. The mean linear intercept (MLI) and mean alveolar number (MAN) were observed in fetal lung tissue at E21.5 following hematoxylin and eosin staining. E13.5 fetal lungs were cultured for $96 \mathrm{~h}$ in serum-free medium and branch development was observed under a microscope. The gene and protein expression levels of EPHB4 and EFNB2 were assessed by reverse transcription-quantitative polymerase chain reaction analysis, and immunoblotting and immunohistochemistry, respectively. The fetal rat lungs were treated with EFNB2 and the activity of key signaling pathways was assessed. The lung index (lung weight/body weight) at E21.5 was significantly lower in the $\mathrm{CDH}$ rats, compared with that in the control fetal rats. The MLI and MAN were also lower in the CDH group. The number of lung terminal buds at E13.5 (embryonic stage), and the lung-explant perimeter and surface were all smaller in the CDH group rats than in the control group at the same age. Pulmonary hypoplasia was observed following $96 \mathrm{~h}$ of in vitro culture. No significant differences were found in the expression levels of EFNB2 and EPHB4 between the
\end{abstract}

Correspondence to: Professor Cai Xia Liu, Department of Gynecology and Obstetrics, Shengjing Hospital Affiliated to China Medical University, 36 Sanhao Street, Heping, Shenyang, Liaoning 110004, P.R. China

E-mail: liucx1716@163.com

Key words: ephrin-B2, ephrin type-B receptor 4, congenital diaphragmatic hernia, lung development, branching
$\mathrm{CDH}$ and control groups at E13.5 (embryonic stage) or E15.5 (pseudoglandular stage), however, EFNB2 and EPHB4 were significantly upregulated at E17.5 (canalicular stage), and at E19.5 and E21.5 (saccular/alveolar stages). EFNB2 stimulated pulmonary branching and EFNB2 supplementation decreased the activity of p38, c-Jun NH2-terminal kinase, extracellular signal-regulated kinase, and signal transducer and activator of transcription. The $\mathrm{CDH}$ fetal rats developed pulmonary dysplasia at an early stage of fetal pulmonary development. Upregulated expression of EFNB2 and EPHB4 was observed in the rat lung of nitrofen-induced $\mathrm{CDH}$, and the increased expression of EFNB2 promoted rat lung development in the nitrofen-induced $\mathrm{CDH}$ model.

\section{Introduction}

Congenital diaphragmatic hernia (CDH) is a common structural fetal malformation, with an incidence of $\sim 1 / 3,000(1,2)$. According to its anatomical classification, $\mathrm{CDH}$ can be divided into posterolateral (Bochdalek hernia), central diaphragmatic, and anterior diaphragmatic hernias. Anterior diaphragmatic hernias include Morgnani (poststernal and parasternal hernias) and other rare hernias. Posterolateral hernias account for $80-90 \%$ of all CDHs, and include left diaphragmatic hernias $(\sim 85 \%)$, right diaphragmatic hernias $(\sim 10 \%)$, and bilateral diaphragmatic hernias ( $5 \%)$ (3-5). Infants with $\mathrm{CDH}$ have a high mortality rate, with respiratory dysfunction, mainly due to concomitant pulmonary hypoplasia and persistent pulmonary hypertension, being the main cause of mortality in $\mathrm{CDH}$ neonates. Long-term follow-up of patients with $\mathrm{CDH}$ has shown that pulmonary dysplasia can lead to pulmonary diseases in surviving children, including obstructive airway disease and restrictive lung function, and children with $\mathrm{CDH}$ are often prone to develop asthma-related difficulties, with a propensity for pulmonary infections and chronic pulmonary hypertension (6-8).

The present study found that the main pathological lung tissue changes in $\mathrm{CDH}$ were abnormal pulmonary branch development, pulmonary hypoplasia and pulmonary hypertension caused by pulmonary vascular dysplasia (9-11). It has been suggested that fetal lung hypoplasia in patients with $\mathrm{CDH}$ is mainly caused by the abdominal visceral hernia protruding into the chest, resulting in compression of the developing lung. 
However, fetal lung dysplasia occurs prior to the occurrence of diaphragmatic hernia in a CDH rat model (12).

Ephrin (Eph) proteins belong to the superfamily of transmembrane tyrosine kinase receptors and were originally identified in human tumors (13). Eph receptors exhibit the prototypical receptor tyrosine kinase topology, with a multidomain extracellular region that includes the ephrin ligand-binding domain, a single transmembrane segment, and a cytoplasmic region containing the kinase domain. Eph receptors have been divided into class A and B receptors, termed EphA and EphB, based on sequence similarity and their preference for binding a particular subclass of ephrins (14). Ephrin type-B receptor 4 (EPHB4) is the receptor for ephrin-B2 (EFNB2) and Eph family members interact with each other through ligand-receptor interactions, and exert their biological effects by altering intracellular signaling pathways (15). The biological functions of Eph proteins include neural development, axon guidance, synaptogenesis, blood and lymphatic vessel development, skeletal patterning, adult stem cell regeneration, and bone homeostasis $(16,17)$. Among these, Eph family molecules have demonstrated dynamic expression during pulmonary vascular development. The expression of EFNB2 and EPHB4 at the arterial-venous interface may restrict the intermingling of arterial and venous endothelial cells, thereby stimulating the formation of new capillary sprouts. In addition, Eph family receptor-ligand interactions on different cell surfaces can promote vascular assembly and are critical in the differentiation of mesenchymal cells into perivascular supporting cells, which is essential for the maintenance of stable and mature vessels (18). The roles of ephrins and their receptors in vascular development, cell migration indicate potential involvement in lung branching morphogenesis

Based on these facts, it is hypothesized that EFNB2 and EPHB4 may have regulatory roles in $\mathrm{CDH}$ fetal lung development. The aim of the present study was to investigate the pathological changes in the $\mathrm{CDH}$ fetal lung, changes in the expression of EFNB2 and EPHB4, and the mechanism involved in affecting lung development in a nitrofen-induced $\mathrm{CDH}$ rat model.

\section{Materials and methods}

Animals. All procedures/protocols were approved by the Animal Research Committee of China Medical University (Shenyang, Liaoning, China). Experimental Sprague-Dawley, specific pathogen-free (SPF) rats were provided by Liaoning Changsheng Biotechnology Co., Ltd. (Benxi, Liaoning, China), and were fed in the SPF-grade laboratory of the Animal Center at Shengjing Hospital Affiliated to China Medical University. The rats were fed a normal diet and were maintained under a 12/12 h day/night cycle at a temperature of $20-25^{\circ} \mathrm{C}$. Female rats (weight 240-260 g) were divided randomly into two groups, and housed in cages with male rats (260-280 g) at a ratio of 3:1 for mating. The female rats were then fed alone following confirmation of sperm in the vaginal smear on the following day, with 12 o'clock denoted as embryonic day (E)0.5. On E8.5, the experimental group was administered with $100 \mathrm{mg}$ nitrofen (cat. no. N141413; Aladdin, Shanghai, China) by oral gavage (100 mg nitrofen dissolved in $1 \mathrm{ml}$ edible oil), whereas the control group was provided with the same quantity of edible oil. Fetuses were then removed by cesarean section on E13.5, E15.5, E17.5, E19.5, and E21.5, following anesthesia with $1 \%$ pentobarbital intraperitoneal injection. The incidence of $\mathrm{CDH}$ in the fetal rats was $64 \%$. Fetal lung tissue was collected under an anatomical microscope and stored at $-80^{\circ} \mathrm{C}$. Certain lung tissues were fixed in $4 \%$ paraformaldehyde for $24-48 \mathrm{~h}$.

Hematoxylin and eosin $(H \& E)$ staining. The anterior halves of the paraffin-embedded lungs (4- $\mu \mathrm{m}$ sections) were deparaffinized in dimethylbenzene and hydrated by ethanol. Three E21.5 lung tissue slices from each group were selected randomly for $H \& E$ staining; $H \& E$ staining was performed according to the manufacturer's protocol (cat. no. G1120, Solarbio Science \& Technology Co., Ltd., Beijing, China). Five fields (upper, middle, lower, left, and right) in each section were also selected randomly (avoiding large vessels and bronchi), observed under Olympus BX61 light microscope (Olympus Corporation, Tokyo, Japan) at x100 magnification, and images were captured using a digital camera. Cross lines were drawn at the center of each field of view, and the number of alveolar spaces (Ns) intersecting the cross line and the number of alveoli in each visual field $(\mathrm{Na})$ were calculated. The total length of the line (L) and the area of each visual field (S) were also measured. The mean linear intercept (MLI) and mean alveolar number (MAN) in the lung tissues were calculated according to the following formulae: $\mathrm{MLI}=\mathrm{L} / \mathrm{Ns}$ (which reflects the mean alveolar diameter) and $\mathrm{MAN}=\mathrm{Na} / \mathrm{S}$ (which reflects alveolar density). Image analysis was performed using Image Pro-Plus 6.0 (Media Cybernetics, Inc., Washington, DC, USA).

Fetal lung explant cultures. The pregnant rats received an intraperitoneal injection of $1 \%$ pentobarbital on E13.5, and the fetuses were removed by cesarean section. The fetal lung tissue was removed under aseptic conditions, transferred to Costar-Transwell ${ }^{\circledR}$ cells (Corning Incorporated, Corning, NY, USA), and cultured at the air-liquid interface in serum-free DMEM/F12 medium (Invitrogen; Thermo Fisher Scientific, Inc., Waltham, MA, USA) supplemented with $100 \mathrm{U} / \mathrm{ml}$ penicillin and $100 \mu \mathrm{g} / \mathrm{ml}$ streptomycin. The three experimental groups (control, $\mathrm{CDH}$ and $\mathrm{CDH}+\mathrm{EFNB} 2$ groups) comprised eight lungs/group. The explants were placed in humidified incubators at $37^{\circ} \mathrm{C}$ in an atmosphere of air plus $5 \% \mathrm{CO}_{2}$ and cultured for $96 \mathrm{~h}$. In the CDH +EFNB2 group, following $1 \mathrm{~h}$ of incubation, recombinant EFNB2 (cat. no. 496-EB-200; R\&D Systems, Inc., Minneapolis, MN, USA) was added to the lung explants at a final concentration of $0.01 \mu \mathrm{g} / \mathrm{ml}$. The recombinant EFNB2 was added daily. An equal volume of phosphate-buffered saline (PBS) was added to the control group. The medium was replaced at $48 \mathrm{~h}$. Images of the lung explants were captured daily using an inverted phase contrast microscope, and lung buds were counted in the digitized images using Image Pro-Plus software 6.0 (Media Cybernetics, Inc.). At the end of the incubation period, the explants were washed in PBS and stored at $-80^{\circ} \mathrm{C}$ until use.

RNA extraction and reverse transcription-quantitative polymerase chain reaction $(R T-q P C R)$ analysis. Total RNA was extracted from the fetal pulmonary tissues using RNAiso 
Table I. Primer sequences.

\begin{tabular}{lllcc}
\hline Target gene & ID & \multicolumn{1}{c}{ Sequence $\left(5^{\prime}-3^{\prime}\right)$} & Temp $\left({ }^{\circ} \mathrm{C}\right)$ & Size $(\mathrm{bp})$ \\
\hline Rat $\beta$-actin & NM_031144.3 & Forward: GGAGATTACTGCCCTGGCTCCTA & 63.7 & 127 \\
& & Reverse: GACTCATCGTACTCCTGCTTGCTG & 63.7 & \\
Rat EFNB2 & NM_001107328.2 & Forward: GCCTTATTCGCAGGGATTG & 57.4 & 98 \\
& & Reverse: CGTGTGCTGTGGAGAGTGTT & 54.1 & \\
Rat EPHB4 & XM_003751157.4 & Forward: TGAGGTGTGCGATATGAAGC & 55.2 & 108 \\
& & Reverse: CAGGGACAGACATTCCATCA & 57.3 & \\
\hline
\end{tabular}

EFNB2, ephrin-B2; EPHB4, ephrin type-B receptor 4.

Plus extraction reagent (cat. no. 9108; Takara Biotechnology Co., Ltd., Beijing, China) according to the manufacturer's protocol. A PrimeScript ${ }^{\mathrm{TM}}$ RT reagent kit with gDNA Eraser (Perfect Real Time; cat. no. RR047A, Takara Biotechnology Co., Ltd.) was used for reverse transcription. Total RNA $(1 \mu \mathrm{g})$ was reverse transcribed into cDNA for qPCR, which was performed in accordance with the manufacturer's protocol using SYBR ${ }^{\circledR}$ Premix Ex Taq ${ }^{\mathrm{TM}}$ II (Tli RNaseH Plus; cat. no. RR820A; Takara Biotechnology Co., Ltd.) on an Applied Biosystems ${ }^{\circledR} 7500$ Fast Real-Time PCR system. The qPCR system included the following: TB Green Premix Ex Taq II $10 \mu \mathrm{l}$; PCR Forward Primer $(10 \mu \mathrm{M}) 0.8 \mu \mathrm{l}$; PCR Reverse Primer (10 $\mu \mathrm{M}) 0.8 \mu \mathrm{l}$; ROX Reference Dye II (50x) $0.4 \mu \mathrm{l}$; cDNA $2 \mu \mathrm{l}$; $\mathrm{ddH}_{2} \mathrm{O} 6 \mu \mathrm{l}$; Total: $20 \mu \mathrm{l}$. Primer synthesis was performed by Sangon Biotech Co., Ltd. (Shanghai, China), as listed in Table I. The specific conditions were as follows: Stage i) $95^{\circ} \mathrm{C}$ for $30 \mathrm{sec}$; stage ii) $95^{\circ}$ for $3 \mathrm{sec}$, and then $60^{\circ}$ for $30 \mathrm{sec}$, repeating 40 times; stage iii) melt curve establishment. The relative mRNA levels were calculated using the $2^{-\Delta \Delta \mathrm{Cq}}$ method (19) following normalization with the housekeeping gene $\beta$-actin.

Western blot analysis. The fresh frozen lungs were thawed and sonicated, and proteins were isolated using the Mem-PER ${ }^{\mathrm{TM}}$ Plus Membrane Protein Extraction kit (cat. no. 89842; Thermo Fisher Scientific, Inc.). Protein concentrations were measured using the Pierce ${ }^{\mathrm{TM}}$ BCA Protein Assay kit (cat. no. 23225; Thermo Fisher Scientific, Inc.) and diluted with gel-loading buffer (cat. no. P0015; Beyotime Institute of Biotechnology, Shanghai, China) prior to gel loading. Protein (quantity, $50 \mu \mathrm{g}$ ) separation was achieved by gel electrophoresis using 10\% SDS-polyacrylamide gels (cat. no. P0012A; Beyotime Institute of Biotechnology) in SDS running buffer. The proteins were transferred onto polyvinylidene difluoride membranes (EMD Millipore, Billerica, MA, USA) by western blotting. Following western blotting, the membranes were blocked in 5\% non-fat milk for 60 min prior to antibody detection. Primary antibodies against EPHB4 [rabbit polyclonal; cat. no. 20883-1-AP, 1:500 dilution in TRIS-buffered saline with $0.1 \%$ Tween-20 (TBST), ProteinTech Group, Inc., Wuhan, China], EFNB2 (rabbit monoclonal; cat. no. ab150411; 1:500 dilution in TBST; Abcam, Cambridge, MA, USA), $\beta$-actin (mouse monoclonal; cat. no. 60008-1-Ig; 1:4,000 dilution in TBST; ProteinTech Group, Inc.), non-phosphorylated and phosphorylated forms of p38, p44/42 [extracellular signal-regulated kinase (ERK)1/2], c-Jun NH2-terminal kinase (JNK) (cat. nos. 9926 and 9910; 1:1,000 dilution in TBST; Cell Signaling Technology, Inc., Beverly, MA, USA) and non-phosphorylated and phosphorylated forms of signal transducer and activator of transcription (STAT)3 (cat. nos. 12640 and 9131; 1:1,000 dilution in TBST; Cell Signaling Technology, Inc.), were incubated overnight at $4^{\circ} \mathrm{C}$. Following extensive washing with TBST, the membranes were incubated with the following horseradish peroxidase (HRP)-conjugated secondary antibodies for $1.5 \mathrm{~h}$ at room temperature: Anti-rabbit IgG, HRP-linked antibody (cat. no. 7074; Cell Signaling Technology, Inc.) and anti-mouse IgG, HRP-linked antibody (cat. no. 7076; Cell Signaling Technology, Inc.), followed by further extensive washing. Detection was performed using an enhanced chemiluminescence kit SuperSignal ${ }^{\mathrm{TM}}$ West Pico PLUS chemiluminescent substrate (cat. no. 34580; Thermo Fisher Scientific, Inc.). $\beta$-actin was used to control for equal loading and transfer of the samples. The optical density (OD) of the protein bands was analyzed using Quantity One software 4.6.7 (Bio-Rad Laboratories, Inc.). The expression of a target protein was calculated as a percentage of the corresponding $\beta$-actin OD. Experiments were performed at least three times for each antibody.

Immunohistochemistry. The paraffin-embedded lungs (4- $\mu \mathrm{m}$ sections) were deparaffinized in dimethylbenzene and hydrated in ethanol. The tissue preparations were boiled for $7 \mathrm{~min}$ in a microwave oven and then cooled to room temperature. Immunohistochemistry was performed using the streptavidin-peroxidase method (cat. no. SP-9001; SPlink detection kit; OriGene Technologies, Inc., Beijing, China) according to the kit protocol. Rabbit polyclonal anti-EPHB4 antibody was added at $4^{\circ} \mathrm{C}(1: 250$, overnight). Rabbit monoclonal anti-EFNB2 was added at $4^{\circ} \mathrm{C}(1: 200$, overnight). The sections were counterstained with hematoxylin (cat. no. G1080; Solarbio Science $\&$ Technology Co., Ltd.) for 90 sec. Negative controls were performed without primary antibody. Images of 60 slides were captured and semi-quantitative analysis was performed using Image Pro-Plus 6.0 (Media Cybernetics, Inc.).

Statistical analysis. Data are presented as the mean \pm standard error of the mean. Statistical analyses were performed using GraphPad Prism 5 (GraphPad Software, Inc., San Diego, CA, 

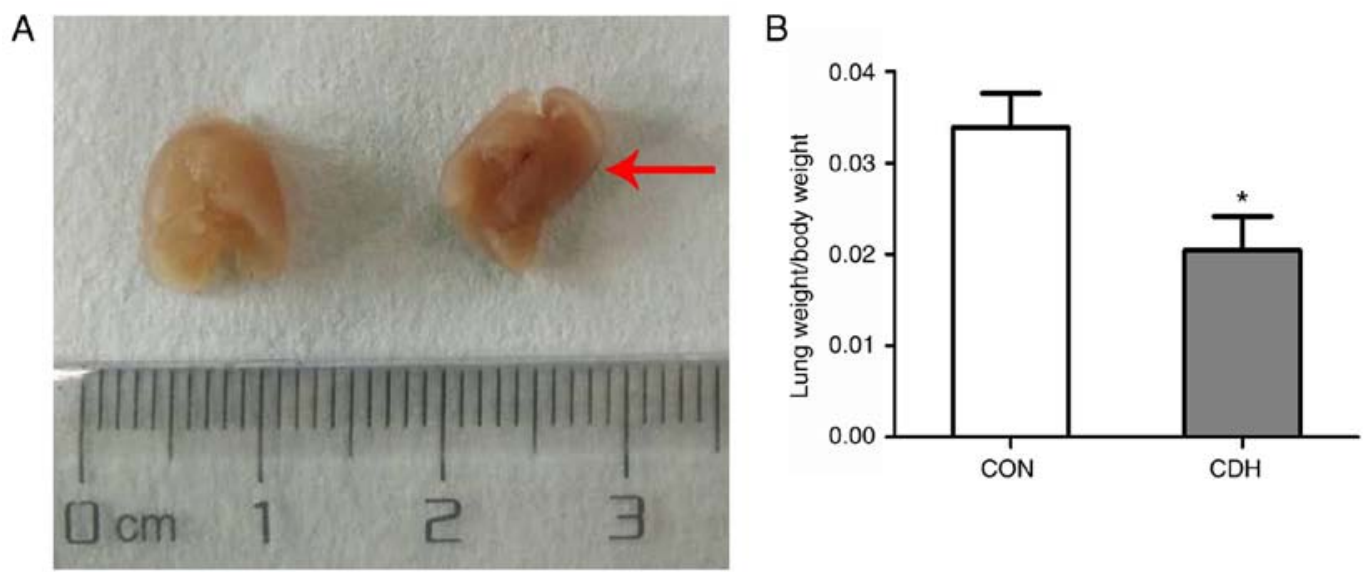

Figure 1. Lung development in CDH fetal rats. (A) E21.5 fetal lung tissue from CON and $\mathrm{CDH}$ rat fetus groups, following fixation in $4 \%$ paraformaldehyde for $48 \mathrm{~h}$. The red arrow indicates the compressed lung in the $\mathrm{CDH}$ group. (B) Fetal rat lung wet weight/body weight was lower in the $\mathrm{CDH}$ group compared with that in the $\mathrm{CON}$ group. Results are presented as the mean \pm standard error of the mean. ${ }^{*} \mathrm{P}<0.05$, vs. CON. CDH, congenital diaphragmatic hernia; $\mathrm{CON}$, control.
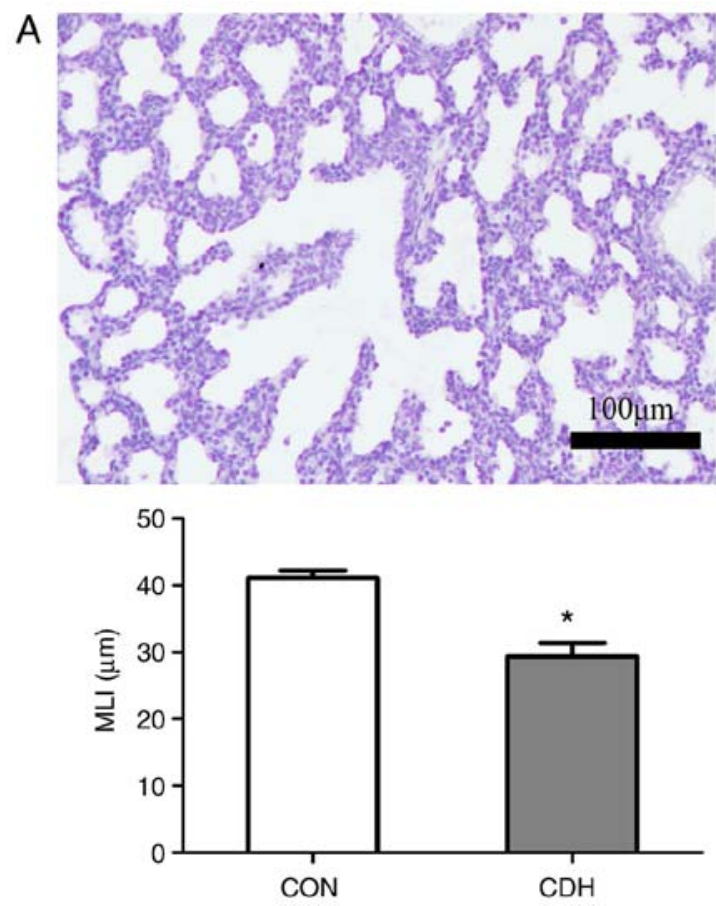

$\mathrm{B}$
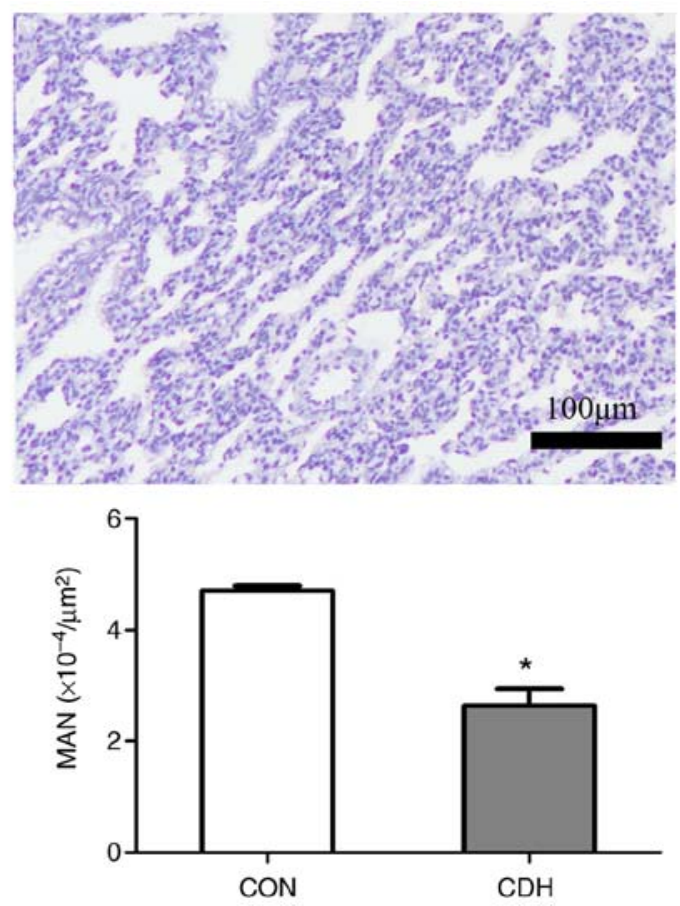

Figure 2. Pathological changes of CDH fetal pulmonary development. (A) H\&E staining of E21.5 fetal lung tissue from CON rat fetuses; (B) H\&E staining of E21.5 fetal lung tissue from CDH rat fetuses. MLI and MAN were significantly lower in the CDH compared with the CON group. Original magnification, $\mathrm{x} 100$, scale bar $=100 \mu \mathrm{m}$. Results are presented as the mean \pm standard error of the mean. ${ }^{*} \mathrm{P}<0.05$, vs. CON. H\&E, hematoxylin and eosin; MLI, Mean linear intercept; MAN, mean alveolar number; $\mathrm{CDH}$, congenital diaphragmatic hernia; CON, control.

USA). Statistical comparison of experimental groups was achieved using unpaired Student's t-test or a one-way analysis of variance. The Student-Newman-Keuls test was used for post hoc analysis. $\mathrm{P}<0.05$ was considered to indicate a statistically significant difference.

\section{Results}

Lung development in $\mathrm{CDH}$ fetal rats. Herniation of the liver and stomach from the abdominal cavity into the left thoracic cavity was observed on E21.5 in the CDH fetal rats, and the volume of lung tissue on the hernia side was significantly smaller in the $\mathrm{CDH}$ fetuses compared with the control fetuses.
The lung index (lung wet weight/body weight) was also significantly lower in the $\mathrm{CDH}$ group compared with that in the control group (Fig. 1A and B).

Pathological changes of CDH fetal pulmonary development. Paraffin sections of lung tissue from E21.5 fetuses were subjected to $H \& E$ staining to reveal morphological changes. The MAN was significantly lower in the $\mathrm{CDH}$ group compared with that in the control group. The MLI in the $\mathrm{CDH}$ group was also significantly reduced compared with that in the control group. These results suggested the existence of pulmonary dysplasia in the $\mathrm{CDH}$ group, 

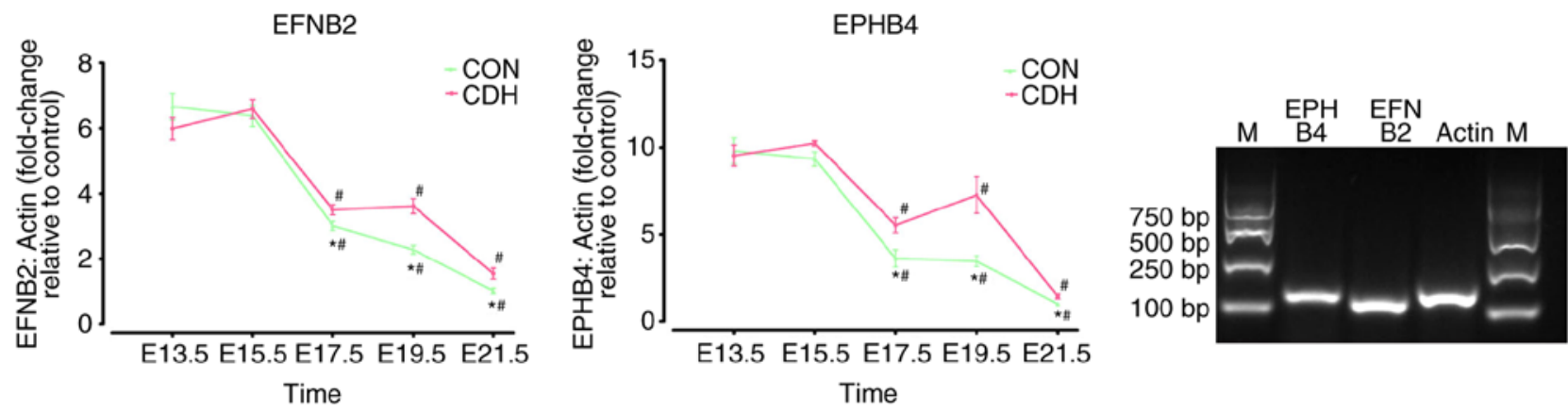

Figure 3. Temporal expression of EFNB2 and EPHB4 in the lungs of the CDH and CON groups. mRNA levels of EFNB2 and EPHB4 in developing lungs were determined by reverse transcription-quantitative polymerase chain reaction analysis and results are expressed relative to the control at E13.5, E15.5, E17.5, E19.5 and E21.5. Specificity of the products were confirmed by visualization on $2 \%$ agarose gels. Results are presented as the mean \pm standard error of the mean. ${ }^{\text {P }}<0.05$, vs. CON at the same time point. ${ }^{*} \mathrm{P}<0.05$, vs. E13.5, inner-group. $\mathrm{CDH}$, congenital diaphragmatic hernia; CON, control; E, embryonic day; EFNB2, ephrin-B2; EPHB4, ephrin type-B receptor 4; M, marker.

with widened alveolar septa and decreased numbers of alveoli (Fig. 2A and B).

Expression of EPHB4 and EFNB2 in lung tissues. The gene expression levels of EFNB2 and EPHB4 were detected in the $\mathrm{CDH}$ and control fetal lung tissues by RT-qPCR analysis at E13.5, E15.5, E17.5, E19.5, and E21.5. No significant differences in expression levels were observed at E13.5 (embryonic stage) or E15.5 (pseudoglandular stage) in the $\mathrm{CDH}$ group compared with the control group, however, EFNB2 and EPHB4 were significantly upregulated at E17.5 (canalicular stage) and E19.5 and E21.5 (saccular/alveolar stages) (Fig. 3). Western blot analysis also showed that the difference in protein expression levels between the two groups were more marked with increasing embryonic age (Fig. 4).

The results of immunohistochemistry showed that the expression of EFNB2 and EPHB4 at different time periods of pregnancy had similarities. EFNB2 was consistently expressed in epithelial cells; however, at E13.5 and E15.5, EFNB2 was also expressed in cells surrounding the epithelium. At E17.5 and E19.5, the expression levels of EFNB2 in the alveolar epithelium and terminal and respiratory bronchioles were higher in the $\mathrm{CDH}$ group than in the control group. At E21.5, EFNB2 was mainly expressed in terminal and respiratory bronchioles, with expression in the $\mathrm{CDH}$ group higher than that in the control group, according to the result of semi-quantitative analysis. EFNB2 was not detected in vascular smooth muscle cells, whereas weak expression was observed in the endothelial cells (Fig. 5A).

In a similar manner, EPHB4 was expressed in less differentiated cells surrounding the epithelium at E13.5 and E15.5. Unlike EFNB2, EPHB4 was expressed in the alveolar epithelium during late lung development, although no expression was detected in the vascular smooth muscle cells (Fig. 5B).

Fetal lung culture. The lung tissues were extracted from the $\mathrm{CDH}$ and control fetal rats on E13.5 and cultured in vitro. The number of terminal lung buds, and the lung tissue explant perimeter and surface were measured at $0,24,48,72$ and $96 \mathrm{~h}$ in the two groups, and these were significantly lower in the $\mathrm{CDH}$
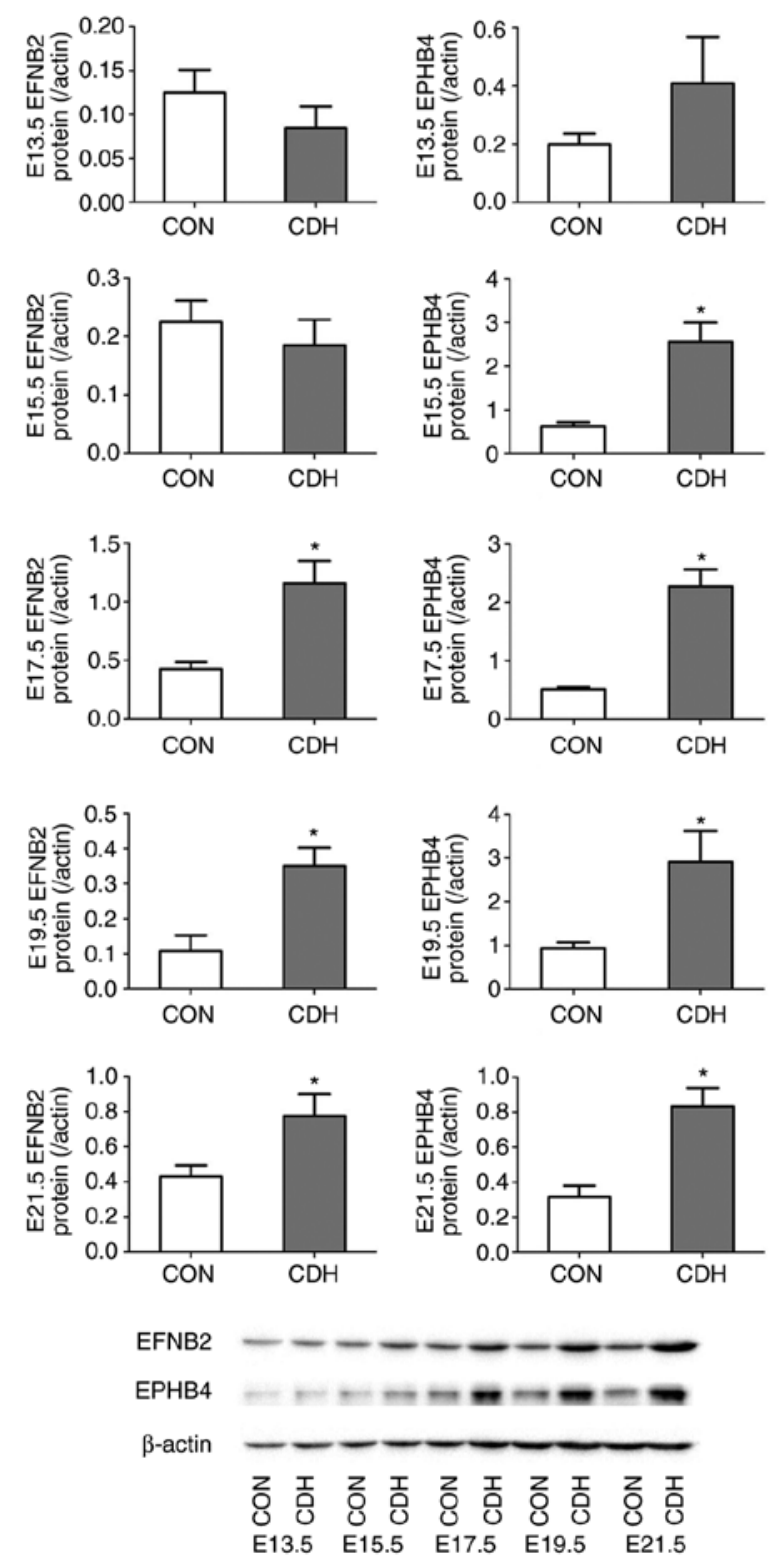

Figure 4. Representative immunoblots and densitometric analysis of expression of EFNB2 and EPHB4 proteins in the fetal lungs at E13.5, E15.5, E17.5, E19.5 and E21.5. Results were normalized relative to the expression of $\beta$-actin and are presented as the mean \pm standard error of the mean. ${ }^{*} \mathrm{P}<0.05$, vs. CON at the same time point. $\mathrm{CDH}$, congenital diaphragmatic hernia; $\mathrm{CON}$, control; E, embryonic day; EFNB2, ephrin-B2; EPHB4, ephrin type-B receptor 4. 
A

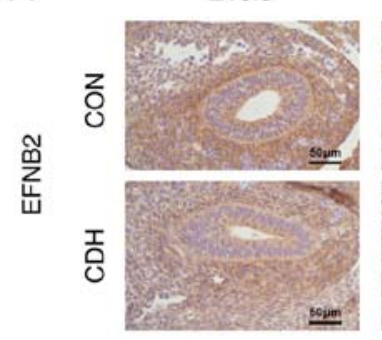

B

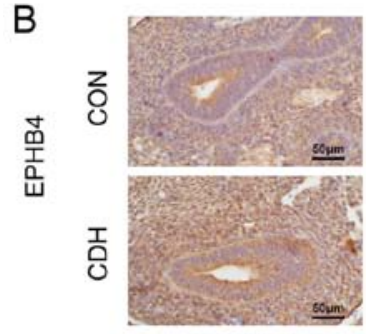

E15.5
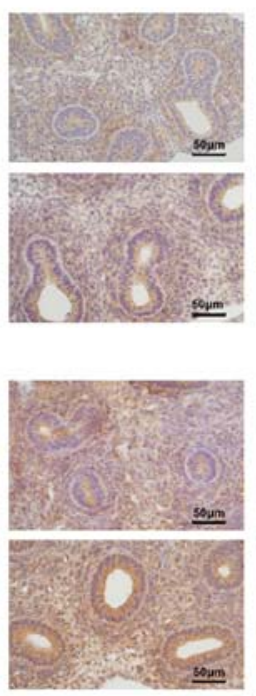

EFNB2

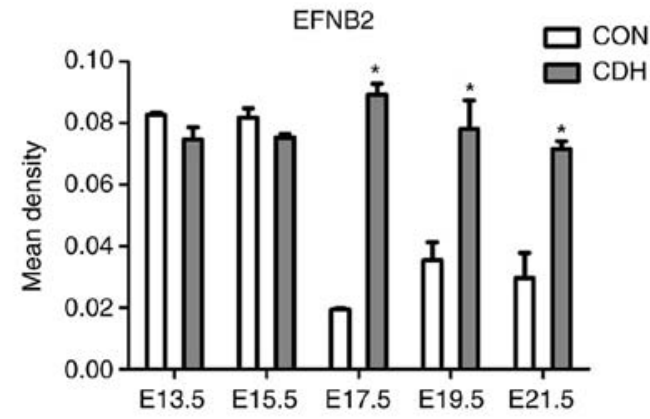

$\mathrm{E} 17.5$
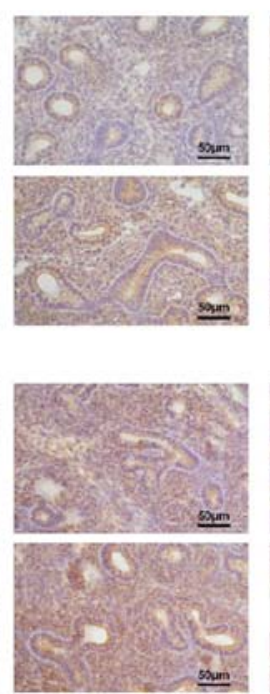

E19.5
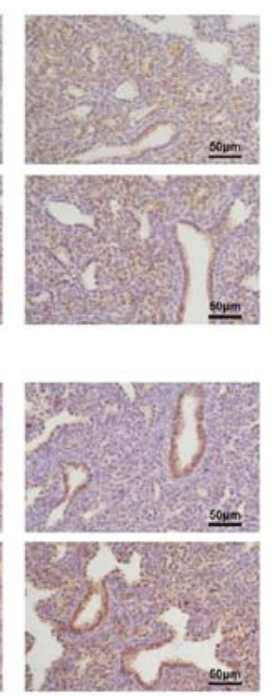

E21.5
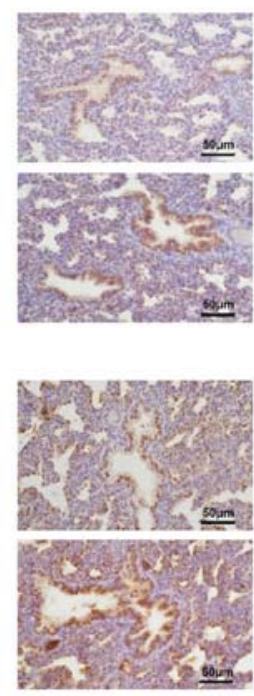

EPHB4

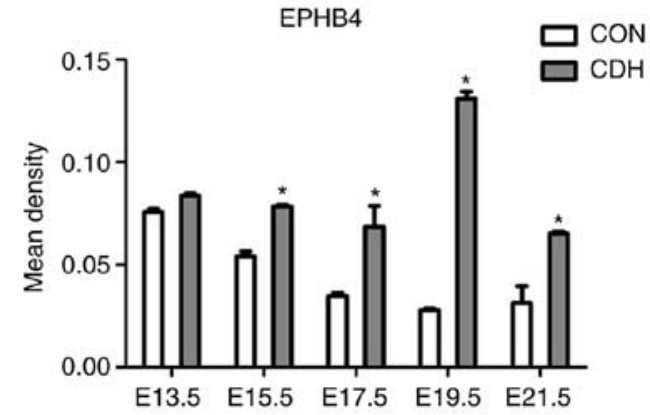

Figure 5. Expression of EFNB2 and EPHB4. Representative photomicrographs of IHC staining for (A) EFNB2 and (B) EPHB4 in the lung sections from CON and $\mathrm{CDH}$ groups at five gestational stages: E13.5, E15.5, E17.5, E19.5 and E21.5 days. EFNB2 and receptor EPHB4 exhibited marked epithelial expression. In the semi-quantitative analysis, at E17.5, E19.5 and E21.5, the expression of EFNB2 and EPHB4 was higher in the CDH group than in the CON group $\left({ }^{*} \mathrm{P}<0.05\right.$ vs. $\mathrm{CON}$ at the same time point). Original magnification, $\mathrm{x} 400$, scale bar=50 $\mu \mathrm{m}$. CDH, congenital diaphragmatic hernia; CON, control; E, embryonic day; EFNB2, ephrin-B2; EPHB4, ephrin type-B receptor 4.

group compared with those in the control group (Fig. 6A-C). Significant differences in all three parameters remained following $96 \mathrm{~h}$ of culture (Fig. 6D and E).

Effect of EFNB2 on fetal lung morphogenesis in rats. To clarify the role of EFNB2 in the development of congenital diaphragmatic hernia in fetal lungs, functional experiments were performed using cultured lung tissue in vitro. Recombinant EFNB2 (final concentration $0.01 \mu \mathrm{g} / \mathrm{ml}$ ) was added to the medium daily and images of the lung tissues were captured every $24 \mathrm{~h}$ (Fig. 6F). It was found that, following the addition of EFNB2 to fetal lung tissue, the development of lung branches was improved, compared with that in the control group at 24, 48, 72, and 96 h (Fig. 6A). At 72 and 96 h of culture, there was a significant difference in the lung tissue area of the two groups (Fig. 6B), and following 48, 72 , and $96 \mathrm{~h}$ of incubation, there was a significant difference in lung tissue circumference between the two groups (Fig. 6C).

EFNB2 influences the phosphorylated forms of P38, ERK, JNK and STAT3. At present, the mechanism of lung development remains to be fully elucidated. To clarify the mechanism involving the effects of EFNB2 on the development of lung branch tissue during congenital diaphragmatic hernia development, western blot analysis was performed on the cultured lung tissues.

Samples from the $\mathrm{CDH}$ and $\mathrm{CDH}+\mathrm{EFNB} 2$ groups were mixed ( $n=10 /$ each group), and changes in the phosphorylation levels of P38, ERK, JNK, and STAT3 were determined. The results showed that EFNB2 treatment inactivated the P38, ERK, JNK, and STAT signaling pathways in the fetal lung explants (Fig. 7A and B).

\section{Discussion}

$\mathrm{CDH}$ is a common congenital anomaly in which an abdominal visceral hernia is caused by diaphragmatic or muscular insufficiency, potentially leading to pulmonary hypoplasia and pulmonary hypertension. Including the effects of induced labor, the mortality rate of $\mathrm{CDH}$ is at least $50 \%$ (20). The majority of CDHs are left-Bochdaleck type diaphragmatic hernias. Although rapid developments in maternal fetal medicine and surgical techniques have enabled resolution of the problem of lung tissue oppression by surgery following birth, pulmonary hypoplasia and pulmonary hypertension caused by abnormal intrauterine lung development remain the main causes of mortality in children with $\mathrm{CDH}$. The quality of life of those surviving 


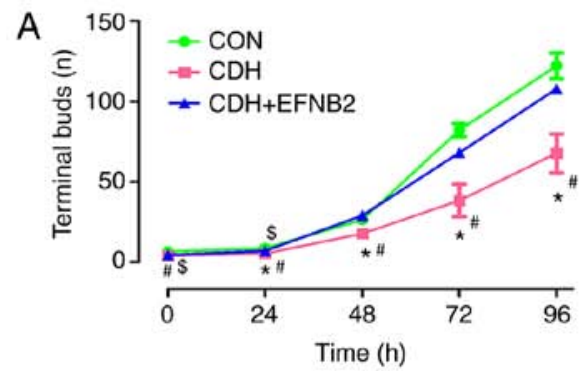

B

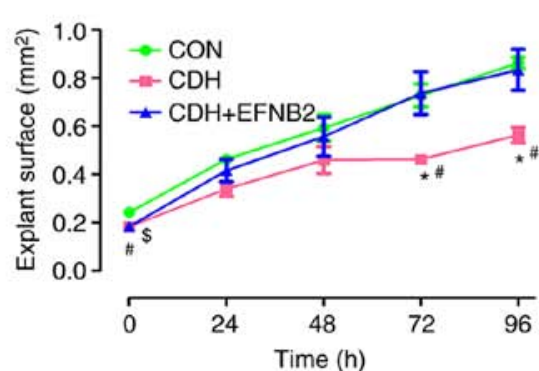

C

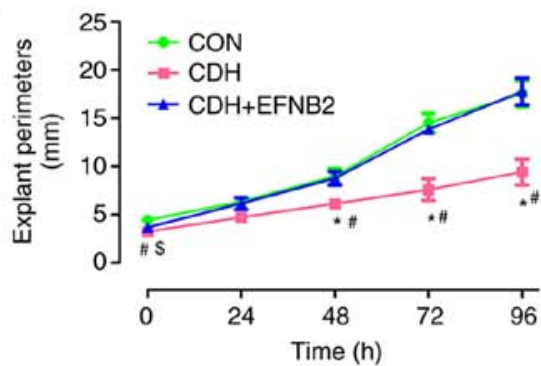

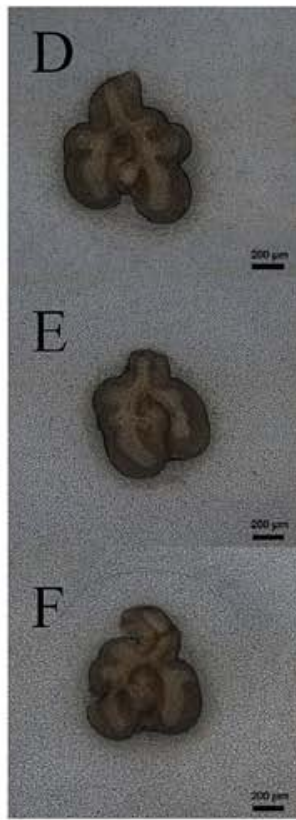

$\mathrm{Oh}$
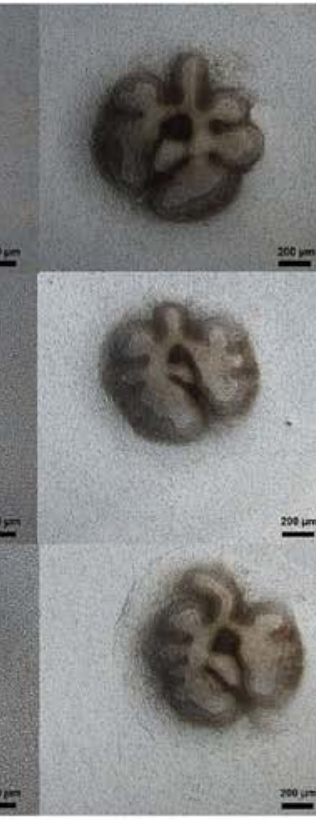

$24 \mathrm{~h}$

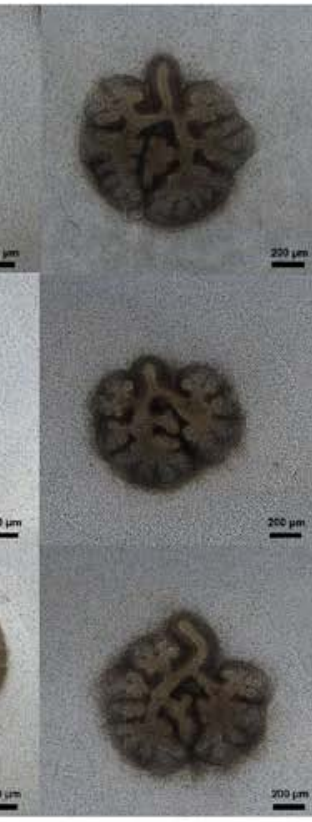

$48 \mathrm{~h}$

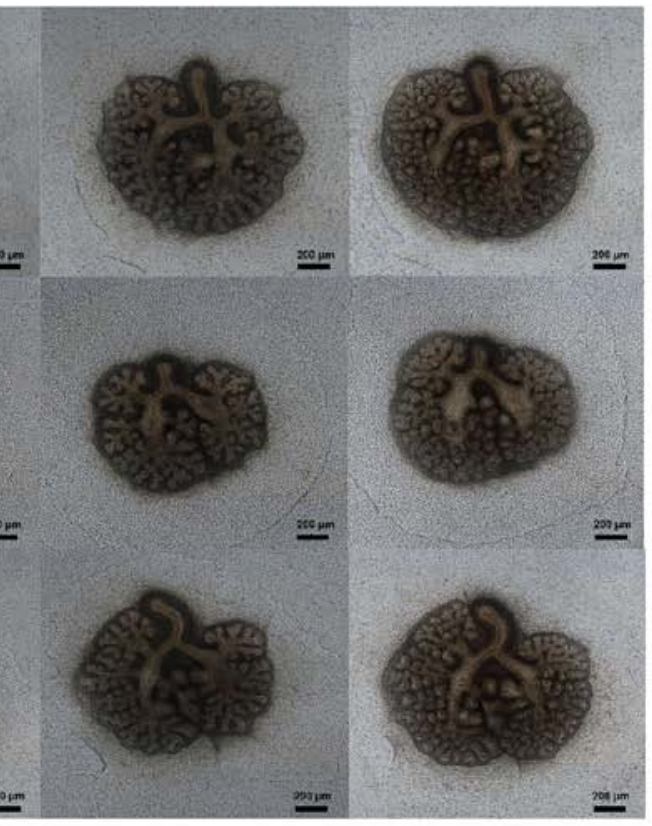

$72 \mathrm{~h}$
$96 \mathrm{~h}$

Figure 6. Fetal lung explant cultures in vitro. (A) Terminal bud count, (B) explant surface, and (C) explant perimeter were significantly smaller in the E13.5 $\mathrm{CDH}$ group than in the $\mathrm{CON}$ group $\left({ }^{\#} \mathrm{P}<0.05\right.$, vs. $\mathrm{CON}$ at the same time point). Lung tissue development in the $\mathrm{CDH}$ group lagged behind that in the $\mathrm{CON}$ group by $96 \mathrm{~h}$ of culture. Lung explants of the (D) CON group (E) CDH group, and (F) CDH+EFNB2 group at 0, 24, 48, 72 and $96 \mathrm{~h}$. EFNB2 supplementation promoted branching of rat fetal lung explants. E13.5 lungs were treated with EFNB2 recombinant protein $(0.01 \mu \mathrm{g} / \mathrm{ml})$ daily. Terminal buds counts, explant surface, and perimeter were significantly increased in the EFNB2-treated group, compared with the CDH group. Compared with the untreated lung, EFNB2 treatment markedly increased airway branching morphogenesis, terminal bud count, explant surface, and explant perimeter at $96 \mathrm{~h}$ (" $\mathrm{P}<0.05, \mathrm{CDH}, \mathrm{vs}$. $\mathrm{CDH}+\mathrm{EFNB} 2$ at the same time point). After $96 \mathrm{~h}$ in vitro, no significant differences in terminal bud count, explant surface, or explant perimeter were observed between the $\mathrm{CDH}+\mathrm{EFNB} 2$ group and $\mathrm{CON}$ group $\left({ }^{\$} \mathrm{P}<0.05, \mathrm{CDH}+\mathrm{EFNB} 2\right.$, vs. $\mathrm{CON}$ at the same time point). Results are presented as the mean \pm standard error of the mean. Original magnification, $\mathrm{x} 40$, scale bar $=200 \mu \mathrm{m}$ (all images at same magnification). $\mathrm{CDH}$, congenital diaphragmatic hernia; $\mathrm{CON}$, control; E13.5, embryonic day 13.5; EFNB2, ephrin-B2.

$\mathrm{CDH}$ has attracted increasing attention, with children who survive $\mathrm{CDH}$ being particularly prone to lung diseases, including chronic pulmonary infection and obstructive pulmonary disease $(3,6-8)$.

Lung development is influenced by several factors, including genetic and environmental factors, involving complex regulatory mechanisms. Rat lung development is divided into five distinct stages: Embryonic stage (E9.5-E14), pseudoglandular stage (E14-E16.5), canalicular stage (E16.5-E17.5), and saccular and alveolar stages (>E17.5) (21). Various growth factors (sonic hedgehog and fibroblast growth factors, particularly fibroblast growth factor 10), transcription factors (hepatocyte nuclear factor-3, and Hox and Gli genes), and signaling pathways, [vascular endothelial growth factor (VEGF) and Ephrin/Eph pathways], have important roles in lung development (22-26).

EFNB2 and EPHB4 are important regulators of pulmonary branch and vascular development. EFNB2 has been shown to control VEGF-induced angiogenesis and 
A
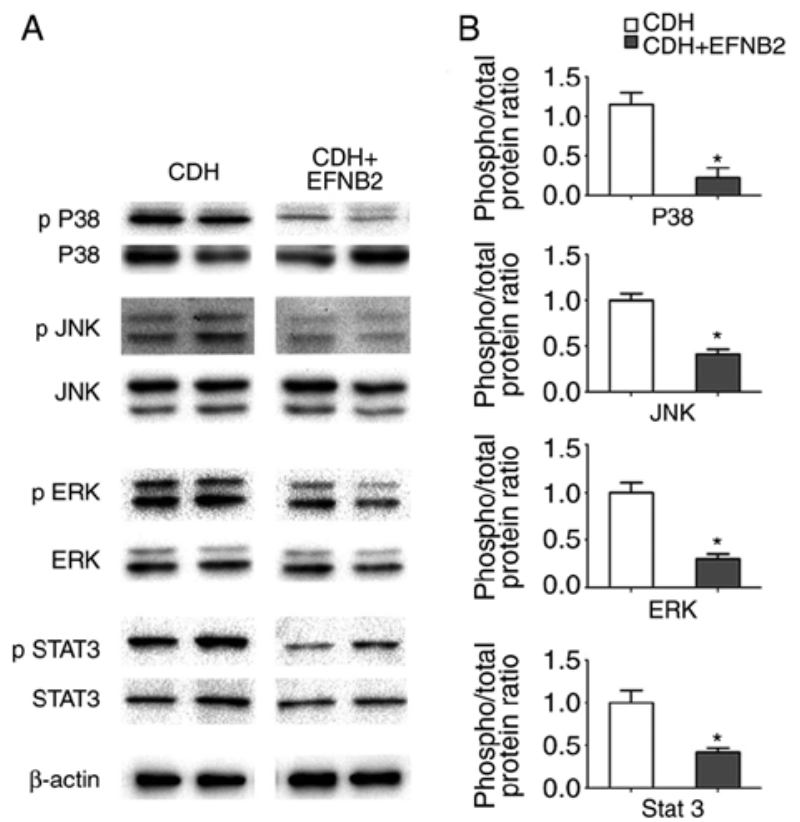

Figure 7. Analysis of the intracellular signaling pathways that the mediate effects of EFNB2 in lung morphogenesis. (A) Representative immunoblots and densitometric analysis of p38, JNK, ERK and STAT3, and p-p38, p JNK, p ERK and p STAT3 in CDH- and EFNB2-treated lung explants (fetal lung explant cultures in vitro for $96 \mathrm{~h}$ ). Results were normalized relative to the expression of $\beta$-actin. (B) Semi-quantitative analysis of phosphorylated forms of intracellular signaling pathways that mediate lung growth. EFNB2 caused a significant decrease in p38, JNK, ERK and STAT3 signaling activity. The activity of intracellular signaling pathways was measured by the ratio between phosphorylated protein and total protein. All data were normalized against the $\mathrm{CDH}$ group. ${ }^{*} \mathrm{P}<0.05$, vs. $\mathrm{CDH}$. EFNB2, ephrin-B2; EPHB4, ephrin type-B receptor 4; $\mathrm{CDH}$, congenital diaphragmatic hernia; CON, control; JNK, c-Jun NH2-terminal kinase; ERK, extracellular signal-regulated kinase; STAT3, signal transducer and activator of transcription 3; p, phosphorylated.

lymphangiogenesis (27). A previous study showed that alveolar development was inhibited when the expression of EFNB2 was inhibited by small interfering RNA in newborn rats in vivo. In a rat model of high-pressure oxygen-induced lung injury, EFNB2 preserved alveolar epithelial cell viability in oxygen, decreased oxygen-induced alveolar epithelial cell apoptosis, and accelerated alveolar epithelial cell wound healing, which had a protective effect in alveolar development. An in vitro study showed that the intranasal administration of EFNB2 inhibited apoptosis in rat alveolar epithelial cells (28). Furthermore, intracellular pathways downstream of EFNB2 were inactivated in EFNB2 mutants, thus mediating distal lung dysplasia and alveolar crest formation. In addition, EFNB2 reverse signaling reduced distal lung compliance by increasing the deposition of $\alpha 5 \beta 1$ integrin-mediated fibronectin (29).

In the present study, the structure of fetal lung tissue in a nitrofen-induced rat model of $\mathrm{CDH}$ was examined. Abnormal lung structure was observed in full-term CDH fetal rats (E21.5), with a reduced alveolar MLI, and reduced numbers of alveoli. The number of lung tissue terminal buds, and the lung tissue explant surface and perimeter were also reduced in the $\mathrm{CDH}$ fetuses compared with the control rat fetuses at E13.5, and lung tissue development continued to lag behind the control group at $96 \mathrm{~h}$ of culture. Previous studies have suggested that compression of the developing lung by the abdominal organs during pregnancy is the main cause of lung dysplasia, however, the development of $\mathrm{CDH}$ lung tissue was delayed even in vitro in the present study. This suggests that molecular defects in the lung tissue itself, in addition to compression, contribute to the development of lung dysplasia.

The present study examined the temporal and spatial changes in the expression of EFNB2 and EPHB4 during lung development. No significant difference was observed in the expression levels of either of these factors between the $\mathrm{CDH}$ and control fetuses during the embryonic (E13.5) and pseudoglandular (E15.5) stages, however, EFNB2 and EPHB4 were significantly upregulated during the canalicular stage (E17.5) and the saccular/alveolar stages (E19.5, E21.5) in the CDH group, with consistent results for mRNA and protein levels. In the present study, lung development in the $\mathrm{CDH}$ fetuses lagged behind that in the controls at E13.5, even following $96 \mathrm{~h}$ of in vitro culture. However, no significant differences were observed in the expression levels of EFNB2 and EPHB4 between the two groups at the embryonic (E13.5) or pseudoglandular (E15.5) stages, possibly due the effect of the hernia-induced compression into the chest cavity on the developing lung being not particularly severe during these stages. However, as the pregnancy progresses, the lung volume increases and its compression becomes more marked, resulting in reconstruction of the lung structure and changes in the expression levels of a series of signaling molecules regulating lung development. It was hypothesized that the expression levels EFNB2 and EPHB4 are increased at the canalicular, saccular, and alveolar stages, to provide compensatory promotion of lung branch development.

The EFNB2 molecule was used to elucidate its role in the development of CDH lungs. Compared with ephrin forward signaling, less is known about ephrin reverse signaling, in which the signal initiation factor is EFNB2, or EFNB2 as a key molecule capable of affecting other molecules involved in lung development (15). Therefore, EFNB2 was selected in the present study as a target gene in the functional experiments. When recombinant EFNB2 was added to fetal lung explant cultures in vitro, it not only promoted the development of CDH lung tissue, but also increased the area and perimeter of this tissue. These results demonstrated that the promotion of EFNB2 during lung development in $\mathrm{CDH}$ fetal rats significantly improved hypoplastic lung conditions.

To clarify the mechanism of how EFNB2 affects lung development, the phosphorylation levels of P38, ERK, JNK, and STAT3 were examined. EFNB2 stimulation induced a decrease in the phosphorylated levels of P38, ERK, JNK, and STAT3, indicating a decrease in these intracellular signaling pathways. The regulation of pulmonary branch development is complex, involving interactions among multiple signaling pathways (30-34). The ERK signaling pathway is a classic signaling pathway, and previous studies have suggested that the ERK/mitogen-activated protein kinase (MAPK) pathway is important in tracheal progenitor cell maintenance and differentiation. The ERK/MAPK pathway is also required for the integration of mesenchymal and epithelial signals required for the development of the entire respiratory tract $(35,36)$. DA-Raf-dependent inhibition of the Ras-ERK signaling pathway in type 2 alveolar epithelial cells is required for alveolar formation (37). The JNK pathway is another distinct 
family included in the MAPK signal transduction pathway, which may also be important in lung development. In a previous study of hyperoxia-induced cell death and impaired alveolarization in the developing lung, of a transforming growth factor- $\beta 1$ transgenic mouse model, inhibition of the JNK signaling pathway significantly improved spontaneously impaired alveolarization in room air and decreased mortality on exposure to hyperoxia (38). For the development of lung branches, rat fetal lung explants at E13.5 cultured in the presence of piceatannol, a known STAT3 signaling inhibitor, have been shown to increase in growth in a dose-dependent manner (39). The possible role of the P38, ERK, JNK and STAT3 signaling pathways in lung growth remains an indirect or even a balancing effect, involving the activation of other pathways, or it may simply be contextual. Further investigations are required to determine whether inactivation of this pathway is crucial for the function of EFNB2 in lung development.

In conclusion, the results of the present study suggested that abnormal intrauterine lung branch development may contribute to pulmonary dysplasia in $\mathrm{CDH}$ fetal rats. The increased expression of the angiogenesis factors, EFNB2 and EPHB4, in CDH fetal lung tissues promoted fetal lung development, however, this was insufficient to completely reverse the CDH-related defects. At present, the effects of fetoscopic endotracheal occlusion on the treatment of severe congenital diaphragmatic hernia remain to be fully elucidated, therefore, EFNB2 may be used as a novel target for intrauterine treatment of severe diaphragmatic hernia. Further investigations are required to determine the exact molecular mechanisms involved in $\mathrm{CDH}$ fetal lung branch development, and the ways in which the Eph family factors can compensate for the abnormal regulation of this process.

\section{Acknowledgements}

Not applicable.

\section{Funding}

The present study was supported by The Establishment and Optimization of Common High-risk Fetal Diagnosis and Treatment Technology Standards and Specifications from the National Health and Family Planning Commission, China (grant no. 201402006).

\section{Availability of data and materials}

The datasets used and/or analyzed during the current study are available from the corresponding author on reasonable request.

\section{Authors' contributions}

HL contributed to the study design, data acquisition and analysis and wrote the manuscript; XL and WY were involved in data acquisition. CL was involved in study design and assisted in writing the manuscript. All authors have read and approved the final manuscript.

\section{Ethics approval and consent to participate}

All procedures performed in experiments involving animals were in accordance with the Animal Research Committee of China Medical University.

\section{Patient consent for publication}

Not applicable.

\section{Competing interests}

The authors declare that they have no competing interests.

\section{References}

1. Torfs CP, Curry CJ, Bateson TF and Honoré LH: A population-based study of congenital diaphragmatic hernia. Teratology 46: 555-565, 1992.

2. Skari H, Bjornland K, Haugen G, Egeland T and Emblem R: Congenital diaphragmatic hernia: A meta-analysis of mortality factors. J Pediatr Surg 35: 1187-1197, 2000.

3. Pober BR, Russell MK and Ackerman KG: Congenital Diaphragmatic Hernia Overview.

4. van Loenhout RB, Tibboel D, Post M and Keijzer R: Congenital diaphragmatic hernia: Comparison of animal models and relevance to the human situation. Neonatology 96: 137-149, 2009.

5. Pober BR: Overview of epidemiology, genetics, birth defects, and chromosome abnormalities associated with CDH. Am J Med Genet C Semin Med Genet 145C: 158-171, 2007.

6. Peetsold MG, Heij HA, Kneepkens CM, Nagelkerke AF, Huisman $\mathrm{J}$ and Gemke RJ: The long-term follow-up of patients with a congenital diaphragmatic hernia: A broad spectrum of morbidity. Pediatr Surg Int 25: 1-17, 2009.

7. Muratore CS, Kharasch V, Lund DP, Sheils C, Friedman S, Brown C, Utter S, Jaksic T and Wilson JM: Pulmonary morbidity in 100 survivors of congenital diaphragmatic hernia monitored in a multidisciplinary clinic. J Pediatr Surg 36: 133-140, 2001.

8. Badillo A and Gingalewski C: Congenital diaphragmatic hernia: Treatment and outcomes. Semin Perinatol 38: 92-96, 2014.

9. Bargy F, Beaudoin S and Barbet P: Fetal lung growth in congenital diaphragmatic hernia. Fetal Diagn Ther 21: 39-44, 2006.

10. Acker SN, Mandell EW, Sims-Lucas S, Gien J, Abman SH and Galambos C: Histologic identification of prominent intrapulmonary anastomotic vessels in severe congenital diaphragmatic hernia. J Pediatr 166: 178-183, 2015.

11. Sluiter I, Reiss I, Kraemer U, Krijger Rd, Tibboel D and Rottier RJ: Vascular abnormalities in human newborns with pulmonary hypertension. Expert Rev Respir Med 5: 245-256, 2011.

12. Guilbert TW, Gebb SA and Shannon JM: Lung hypoplasia in the nitrofen model of congenital diaphragmatic hernia occurs early in development. Am J Physiol Lung Cell Mol Physiol 279: L1159-L1171, 2000.

13. Hirai H, Maru Y, Hagiwara K, Nishida J and Takaku F: A novel putative tyrosine kinase receptor encoded by the eph gene. Science 238: 1717-1720, 1987.

14. Pasquale EB: Eph-ephrin bidirectional signaling in physiology and disease. Cell 133: 38-52, 2008.

15. Klein R: Eph/ephrin signalling during development. Development 139: 4105-4109, 2012.

16. Kania A and Klein R: Mechanisms of ephrin-eph signalling in development, physiology and disease. Nat Rev Mol Cell Biol 17: 240-256, 2016.

17. Lisabeth EM, Falivelli G and Pasquale EB: Eph receptor signaling and ephrins. Cold Spring Harb Perspect Biol 5: a009159, 2013.

18. Cheng N, Brantley DM and Chen J: The ephrins and eph receptors in angiogenesis. Cytokine Growth Factor Rev 13: 75-85, 2002.

19. Livak KJ and Schmittgen TD: Analysis of relative gene expression data using real-time quantitative PCR and the $2^{-\Delta \Delta C_{\mathrm{T}}}$ method. Methods 25: 402-408, 2001. 
20. Downard CD, Jaksic T, Garza JJ, Dzakovic A, Nemes L, Jennings RW and Wilson JM: Analysis of an improved survival rate for congenital diaphragmatic hernia. J Pediatr Surg 38: 729-732, 2003.

21. Kinane TB: Lung development and implications for hypoplasia found in congenital diaphragmatic hernia. Am J Med Genet C Semin Med Genet 145C: 117-124, 2007.

22. Warburton D, Schwarz M, Tefft D, Flores-Delgado G, Anderson KD and Cardoso WV: The molecular basis of lung morphogenesis. Mech Dev 92: 55-81, 2000.

23. Cardoso WV: Molecular regulation of lung development. Annu Rev Physiol 63: 471-494, 2001.

24. Groenman F, Unger S and Post M: The molecular basis for abnormal Human lung development. Biol Neonate 87: 164-177, 2005.

25. Cardoso WV and Lü J: Regulation of early lung morphogenesis: Questions, facts and controversies. Development 133: 1611-1124, 2006.

26. Ware LB and Matthay MA: Keratinocyte and hepatocyte growth factors in the lung: Roles in lung development inflammation and repair. Am J Physiol Lung Cell Mol Physiol 282: L924-L940, 2002.

27. Wang Y, Nakayama M, Pitulescu ME, Schmidt TS Bochenek ML, Sakakibara A, Adams S, Davy A, Deutsch U, Lüthi U, et al: Ephrin-B2 controls VEGF-induced angiogenesis and lymphangiogenesis. Nature 465: 483-486, 2010.

28. Vadivel A, van Haaften T, Alphonse RS, Rey-Parra GJ, Ionescu L, Haromy A, Eaton F, Michelakis E and Thébaud B: Critical role of the axonal guidance cue EphrinB2 in lung growth, angiogenesis, and repair. Am J Respir Crit Care Med 185: 564-574, 2012.

29. Bennett KM, Afanador MD, Lal CV, Xu H, Persad E, Legan SK Chenaux G, Dellinger M, Savani RC, Dravis C, et al: Ephrin-B2 reverse signaling increases $\alpha 5 \beta 1$ integrin mediated fibronectin deposition and reduces distal lung compliance. Am J Respir Cell Mol Biol 49: 680-687, 2013.
30. Sikkema AH, den Dunnen WF, Hulleman E, van Vuurden DG, Garcia-Manero G, Yang H, Scherpen FJ, Kampen KR, Hoving EW, Kamps EW, et al: EphB2 activity plays a pivotal role in pediatric medulloblastoma cell adhesion and invasion. Neuro Oncol 14: 1125-1135, 2012.

31. Nogueira-Silva C, Piairo P, Carvalho-Dias E, Veiga C, Moura RS and Correia-Pinto J: The role of glycoprotein 130 family of cytokines in fetal rat lung development. PLoS One 8: e67607, 2013.

32. Nogueira-Silva C, Piairo P, Carvalho-Dias E, Peixoto FO, Moura RS and Correia-Pinto J: Leukemia inhibitory factor in rat fetal lung development: Expression and functional studies. PLoS One 7: e30517, 2012.

33. Kling DE, Lorenzo HK, Trbovich AM, Kinane TB, Donahoe PK and Schnitzer JJ: MEK-1/2 inhibition reduces branching morphogenesis and causes mesenchymal cell apoptosis in fetal rat lungs. Am J Physiol Lung Cell Mol Physiol 282: L370-L378, 2002.

34. Piairo P, Moura RS, Nogueira-Silva C and Correia-Pinto J: The apelinergic system in the developing lung: Expression and signaling. Peptides 32: 2474-2483, 2011.

35. Boucherat O, Nadeau V, Bérubé-Simard FA, Charron $\mathrm{J}$ and Jeannotte L: Crucial requirement of ERK/MAPK signaling in respiratory tract development. Development 21: 3197-3211, 2014.

36. Boucherat O, Landry-Truchon K, Aoidi R, Houde N, Nadeau V, Charron J and Jeannotte L: Lung development requires an active ERK/MAPK pathway in the lung mesenchyme. Dev Dyn 246: 72-82, 2017.

This work is licensed under a Creative Commons Attribution-NonCommercial-NoDerivatives 4.0 International (CC BY-NC-ND 4.0) License. 